\title{
Análise de Arranjos de Antenas de Microfita Baseados na Geometria Fractal de Tetra Círculo pelo Método WCIP
}

\author{
Mychael J. Duarte, Valdemir P. Silva Neto e Adaildo G. D'Assunção
}

\begin{abstract}
Resumo-Este trabalho apresenta uma investigação teórica $\mathrm{e}$ experimental sobre as propriedades dos arranjos de antenas de microfita com base no fractal tetra círculo. Uma antena foi analisada com um patch ressoador para as frequências de Bluetooth e Wi-Fi; posteriormente, foram adicionados mais patches idênticos ao longo do eixo horizontal, formando os arranjos analisados. Para o estudo, arranjos com dois e quatro elementos ressonantes foram considerados. Todas as estruturas propostas foram construídas. Resultados como perda de retorno e diagramas de radiação foram obtidos usando o Ansoft HFSS, método WCIP e CST Studio. Os resultados foram comparados e discutidos com as medições dos protótipos. Uma boa concordância entre os resultados medidos e simulados é observada.
\end{abstract}

Palavras-Chave- Arranjos de Antenas, Patch Fractal, Antena de Microfita. Método WCIP.

Abstract - This work presents a theoretical and experimental investigation on the properties of microstrip antenna arrays based on the tetra circle fractal. An antenna was analyzed with a resonator patch for the Bluetooth and $\mathrm{Wi}-\mathrm{Fi}$ frequency, later, more patches identical to the horizontal axis logo were added, forming analyzed arrays. For the study, arrangements with two and four resonating elements. All proposed structures were constructed and. Results such as return loss and radiation diagrams were obtained using the Ansoft HFSS, WCIP Method and CST Studio software and were compared and discussed with the measurements. A good agreement between the measured and simulated results is observed.

Keywords-Antenna Arrays, Fractal Patch, Microstrip Antenna, WCIP Method.

\section{INTRODUÇÃO}

Os sistemas modernos de comunicação sem fio buscam por dispositivos menores e que permitam um grande número de aplicações de serviços com taxas de transmissão e recepção maiores. Um modelo que tem ganhado bastante atenção dos pesquisadores, são os circuitos integrados e planares de microfita, por atenderem as necessidades atuais do mercado [1][2]. Com o crescimento das aplicações e o avanço dos sistemas de comunicação wireless, surge a necessidade do uso de antenas compactas com alto ganho e que atuem em diferentes sistemas, decorrente da variedade de aparelhos de comunicação sem fio portátil, como por exemplo telefones celulares, handsets, palmtops, laptops e dentre outros [1]-[3].

Mychael J. Duarte, Valdemir P. Silva Neto e Adaildo G. D’Assunção, Departamento de Engenharia e Comunicações, Universidade Federal do Rio Grande do Norte, Natal-RN, e-mails: mychael_duarte@hotmail.com; vpraxedes.neto@gmail.com; adaildo@ct.ufrn.br;
Basicamente, uma antena planar de microfita consiste de um patch condutor, que é o elemento radiante, sobre um substrato dielétrico, e um plano de terra. Normalmente, o patch condutor assume diversas geometrias que variam de retângulos, círculos, polígonos regulares, até geometrias mais complexas como fractais ou quase fractais [4].

Estudos já consolidados mostram que uso de antenas em arranjos é uma boa alternativa quando se deseja aumentar o ganho e diminuir a largura de feixe de meia potência, deixando a antena mais diretiva. Para aplicações em sistemas de visada direta que necessitam de ganho elevado, arranjo de antenas é uma solução a ser considerada [4]-[5].

Outro ponto que tem chamado bastante atenção dos pesquisadores no estudo de antenas é a utilização de geometrias fractais em seus patches. Esse fato ocorre pelo comportamento multibanda e a dupla polarização apresentados em alguns estudos de fractais. A geometria fractal apresenta em alguns casos uma redução na largura de banda com o aumento da iteração do fractal, tornando a estrutura mais seletiva [6].

Em [7], foram propostos dois arranjos com elementos baseados em retângulos, um arranjo $5 \times 1$ e outro $5 \times 2$, os arranjos apresentaram um ganho de $8,97 \mathrm{dBi}$ e $12,10 \mathrm{dBi}$, respectivamente. Para o plano $\mathrm{E}, \mathrm{o}$ arranjo $5 \times 1$ teve uma largura de feixe a meia potência de $37^{\circ}$, e o outro arranjo de $27,5^{\circ}$, para o plano $\mathrm{H}$, o primeiro arranjo teve uma largura de feixe de $82,5^{\circ}$ e o segundo de $40^{\circ}$.

Em [2], foi proposto um novo método de obtenção de arranjos de antenas planares para aplicações de banda larga e alto ganho para ondas milimétricas. O elemento trata-se de uma nova estrutura de ressonância acoplada verticalmente de terceira ordem, composto por uma abertura, em formato de U no plano de terra que tem a função de acoplar simultaneamente a linha de alimentação e o patch. Foi analisado um arranjo 4x4, e este obteve uma largura de banda relativa igual a $37 \%$ e $19 \mathrm{dBi}$ de ganho.

Autores propuseram um arranjo de elementos retangulares otimizados com dois, oito e dezesseis elementos [8]. O arranjo com dois elementos teve um ganho de 13,93 $\mathrm{dBi}$, o de oito elementos $15,68 \mathrm{dBi}$ e o de dezesseis elementos $17,7 \mathrm{dBi}$. A frequência de ressonância desse arranjo era próxima de 10,0 $\mathrm{GHz}$.

O método iterativo das ondas (WCIP - Wave Concept Iterative Procedure) é uma formulação iterativa de onda 
completa baseada no processo de reflexão e transmissão de ondas na interface do circuito. Ultimamente, o método WCIP tem sido amplamente utilizado para projetar e caracterizar vários circuitos planares, como antenas de microfita [9], filtros [10] e superfícies seletivas de frequência [11], [12].

O objetivo deste trabalho é realizar a caracterização numérica e estudo de comportamento da resposta em frequência, diagrama de radiação e densidade de corrente, para arranjos de antenas com geometria baseada no fractal tetra círculo no nível dois de iteração. Os resultados numéricos foram calculados usando o método WCIP e simulações nos softwares comerciais ANSYS HFSS e CST Studio. As antenas deste trabalho funcionam para aplicações bluetooth e WI-FI. Todos os protótipos analisados nesse trabalho foram construídos e medidos, apresentando boa concordância entre os resultados simulados e medidos.

\section{CONFIGURAÇÃO DOS ARRANJOS}

Os arranjos de antenas propostos nesse trabalho têm o patch baseado em fractal tetra círculo. Foram propostas quatro estruturas, como está apresentado na Fig.1. As estruturas analisadas consistem em uma antena com um elemento patch, e três estruturas distintas de arranjos.

Todas as estruturas possuem um raio $\mathrm{r}_{\mathrm{a}}$ igual a $12,5 \mathrm{~mm}$, uma espessura de linha de alimentação yL igual a 2,10 mm e um comprimento de linha de alimentação $\mathrm{x}_{\mathrm{L}}$ igual a $11,0 \mathrm{~mm}$.

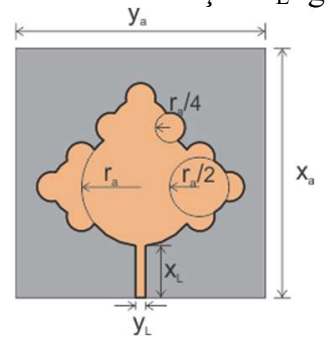

(a)

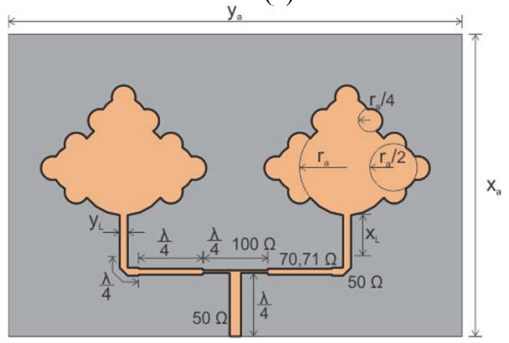

(b)

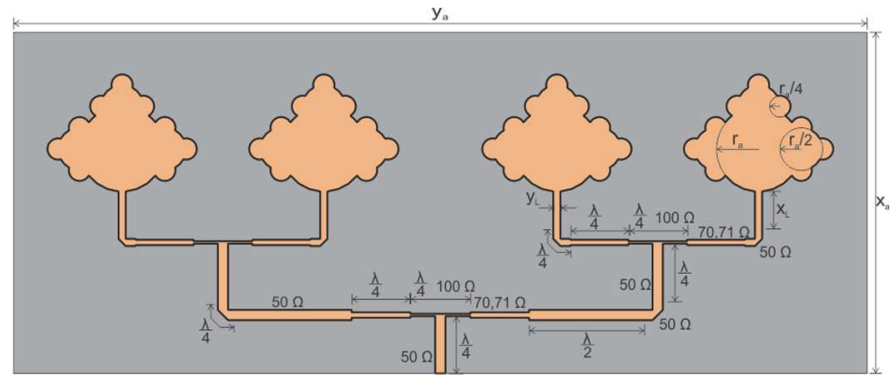

(c)

Fig. 1. Geometrias e arranjos analisados: (a) Arranjo 1x1; (b) Arranjo 1x2; e (c) Arranjo 1x4.
A Fig. 2 mostra a foto dos protótipos construídos. impressos sobre um substrato dielétrico de FR4 de permissividade elétrica relativa igual a 4,4, espessura igual a $1,57 \mathrm{~mm}$, tangente de perdas igual a 0,02 e possuem o plano de terra cheio.

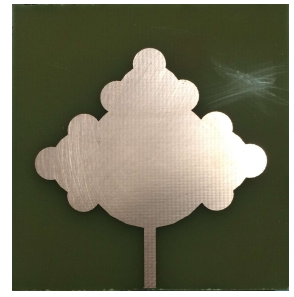

(a)

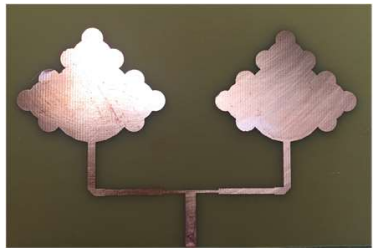

(b)

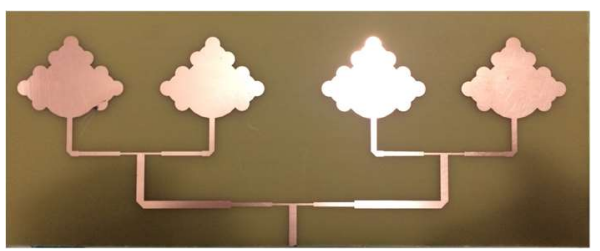

(c)

Fig. 2. Protótipos das antenas: (a) Arranjo 1x1; (b) Arranjo 1x2; e (c) Arranjo $1 \times 4$.

As características de comprimento e impedância para as linhas de alimentação e interconexão nos arranjos foram calculadas para obter casamento de impedância e garantir uma distribuição de corrente uniforme entre os elementos dos arranjos.

\section{FORMULAÇÃO DO MÉTODO WCIP}

O procedimento iterativo do conceito de onda (WCIP) é uma técnica numérica iterativa usada em análises planares de circuitos de micro-ondas [9]-[12]. Ele é baseado na razão de transmissão e reflexão das ondas eletromagnéticas incidente, $\overrightarrow{A_{i}}$, e refletida, $\overrightarrow{B_{i}}$, na interface do circuito que está sendo analisada. Essas equações são dadas por:

$$
\begin{gathered}
\overrightarrow{B_{i}}=\widehat{S} \overrightarrow{A_{i}}+\overrightarrow{A_{0}}, \\
\overrightarrow{A_{i}}=\widehat{\Gamma} \overrightarrow{B_{i}} .
\end{gathered}
$$

As Equações (1) e (2) representam as equações básicas da formulação do WCIP [9],[12]. A equação (1) representa o efeito do circuito de interface na relação entre as ondas $\overrightarrow{A_{i}}$ e $\overrightarrow{B_{i}}$.

O operador de espalhamento $\hat{S}$ leva em consideração a condição de contorno em cada região da interface do circuito [8]. É definido impondo as condições de continuidade do campo eletromagnético em diferentes meios: condutores, dielétricos, cargas e fontes.

A Equação (2) representa a influência do meio em torno do circuito de interface analisado. O operador de reflexão $\widehat{\Gamma}$ é definido no domínio modal e leva em consideração as condições de propagação do meio ao redor do circuito e da interface.

O processo iterativo do método WCIP é definido pela transformação entre os domínios espacial e modal. As equações (1) e (2) são calculadas em cada etapa da iteração até que a convergência seja alcançada. A transformação entre domínios 
espaciais e modais para os componentes de campo é feita usando a transformada de Fourier.

\section{RESUltAdOS E DiscussõES}

A Fig. 3 mostra os gráficos de perda de retorno das comparações entre a respostas simuladas no HFSS, WCIP e no CST, e das medições.

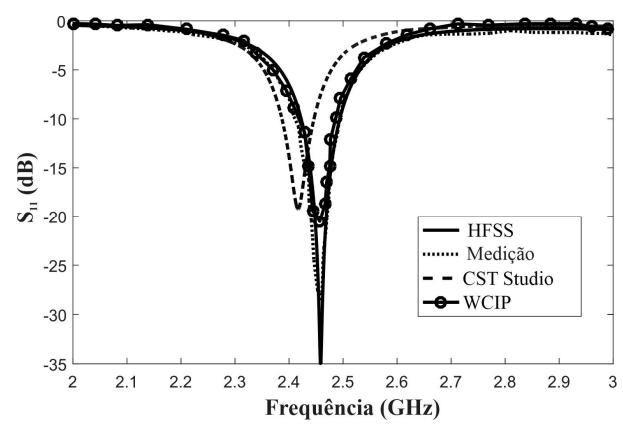

(a)

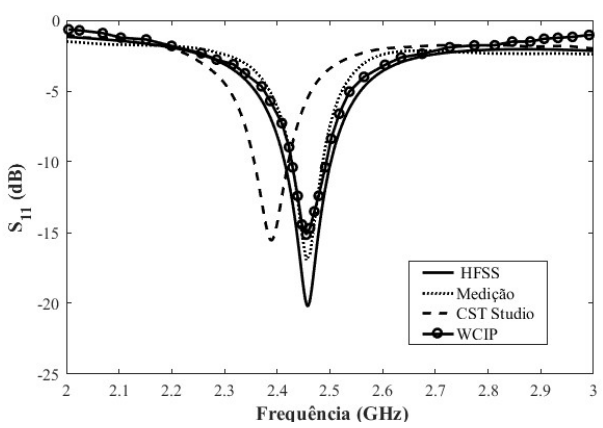

(b)

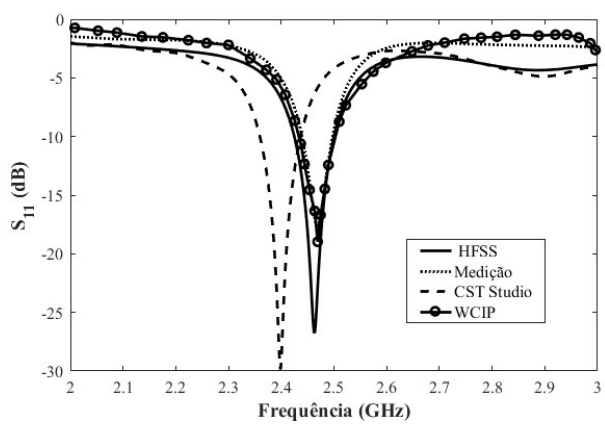

(c)

Fig. 3. Comparação de curvas de perda de retorno simuladas e medidas: (a) Arranjo 1x1; (b) Arranjo 1x2; e (c) Arranjo 1x4.

De acordo com a Fig. 3(a), para a simulação no HFSS a antena com um elemento teve a frequência de ressonância igual a 2,459 GHz, com um $\mathrm{S}_{11}$ igual a $-34,90 \mathrm{~dB}$ e largura de banda relativa de $2,890 \%$. Para a simulação no CST a antena ressoou em 2,416 GHz, com largura de banda relativa igual a 2,525\% e $-19,02 \mathrm{~dB}$ de $\mathrm{S}_{11}$. Na simulação com o WCIP a antena ressoou em $2,457 \mathrm{GHz}$ com $\mathrm{S}_{11}$ igual a $-20,53 \mathrm{~dB}$ e com largura de banda relativa de 3,130\%. Na medição, a antena teve a frequência de ressonância igual a $2,457 \mathrm{GHz}$, com largura de banda relativa de $3,460 \%$ e $-28,52 \mathrm{~dB}$ de $\mathrm{S}_{11}$.
A Fig. 3(b) apresenta a resposta em frequência para o arranjo da Fig. 2(b). De acordo com as curvas, pode-se observar que o considerando simulação no HFSS, este arranjo apresenta uma frequência de ressonância igual a $2,456 \mathrm{GHz}$, com $\mathrm{S}_{11}$ igual a 20,07 dB e largura de banda relativa de 3,50\%. Já na simulação do CST, o arranjo ressoou em $2,391 \mathrm{GHz}$, com largura de banda relativa igual a $2,72 \%$ e $\mathrm{S}_{11} \mathrm{em}-15,50 \mathrm{~dB}$. Para a simulação com WCIP a antena ressoou em $2,455 \mathrm{GHz}$ com largura de banda relativa igual a $2,80 \%$ e $-15,14 \mathrm{~dB}$ de $\mathrm{S}_{11}$. Os valores da medição se aproximaram mais da simulação no HFSS, com uma frequência de ressonância em 2,455 GHz, com $\mathrm{S}_{11}$ em -16,94 dB e largura de banda relativa igual a 2,65\%.

A Fig. 3(c), demonstra que para a simulação no HFSS o arranjo 1x4 teve a frequência de ressonância igual a 2,464 GHz, com um $\mathrm{S}_{11}$ igual a $-26,59 \mathrm{~dB}$ e largura de banda relativa de 3,04 \%. Para a simulação no CST o arranjo ressoou em 2,400 GHz, com largura de banda relativa igual a 3,21\% e -29,80 dB de $\mathrm{S}_{11}$. $\mathrm{Na}$ simulação com WCIP a estrutura ressoou em $2,469 \mathrm{GHz}$ com $\mathrm{S}_{11}$ igual a $-18,98 \mathrm{~dB}$ e largura de banda relativa igual a $2,95 \%$. $\mathrm{Na}$ medição, o arranjo teve a frequência de ressonância igual a $2,465 \mathrm{GHz}$, com largura de banda relativa de 2,43\% e -17,35 dB de perda de retorno.

A Tabela I resume os valores das comparações entre simulações e medições.

TABLE I. COMPARAÇÃO DOS RESULTADOS SIMULADOS E MEDIDOS PARA A RESSONANCIA DAS ANTENAS

\begin{tabular}{|c|c|c|c|c|}
\hline Estrutura & Análise & $\mathbf{f}_{\mathbf{r}}(\mathbf{G H z})$ & BW (\%) & $\mathrm{S}_{11}(\mathrm{~dB})$ \\
\hline \multirow{4}{*}{$\begin{array}{c}\text { Arranjo } \\
\mathbf{1 x 1}\end{array}$} & HFSS & 2,459 & 2,890 & $-34,90$ \\
\hline & CST & 2,416 & 2,525 & $-19,02$ \\
\hline & WCIP & 2,457 & 3,13 & $-20,53$ \\
\hline & Medição & 2,457 & 3,46 & $-28,52$ \\
\hline \multirow{4}{*}{$\begin{array}{c}\text { Arranjo } \\
1 \times 2\end{array}$} & HFSS & 2,460 & 3,50 & $-20,07$ \\
\hline & CST & 2,391 & 2,72 & $-15,50$ \\
\hline & WCIP & 2,455 & 2,80 & $-15,14$ \\
\hline & Medição & 2,455 & 2,65 & $-16,94$ \\
\hline \multirow{4}{*}{$\begin{array}{c}\text { Arranjo } \\
\mathbf{1 x 4}\end{array}$} & HFSS & 2,464 & 3,04 & $-26,59$ \\
\hline & CST & 2,400 & 3,21 & $-29,80$ \\
\hline & WCIP & 2,469 & 2,95 & $-18,98$ \\
\hline & Medição & 2,465 & 2,43 & $-17,35$ \\
\hline
\end{tabular}

A Fig. 4 apresenta um comparativo das simulações de como se comporta a radiação das antenas em função dos ganhos, nos diagramas de radiação $2 \mathrm{D}$ (duas dimensões) dos planos x-z e y$\mathrm{z}$, em dBi. 


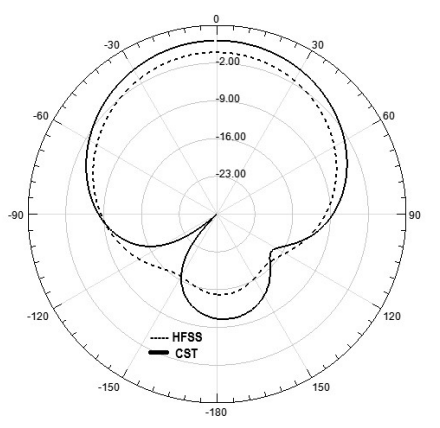

Plano y-z

(a)

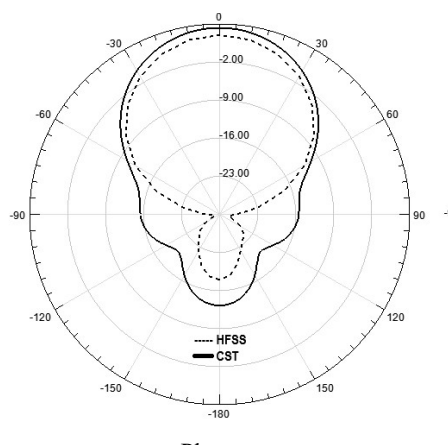

Plano y-z

(b)

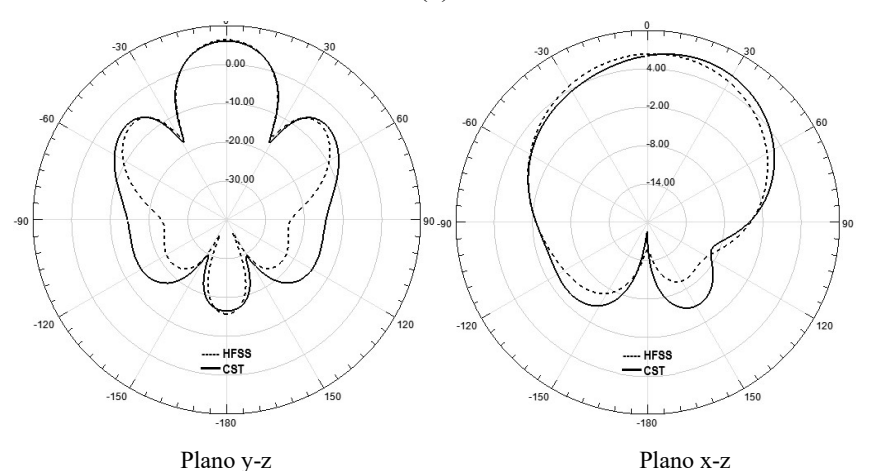

(c)

Fig. 4. Diagramas de radiação simulados 2D: (a) Arranjo 1x1; (b) Arranjo 1x2; e (c) Arranjo 1x4.

De acordo com a Fig. 4(a) nota-se que a antena com um elemento se comporta como direcional e tem seu ganho máximo na direção de Broadside, perpendicular ao plano da antena. $\mathrm{Na}$ simulação do HFSS, a antena apresentou um ganho de $0 \mathrm{~dB}$ nos planos $\mathrm{x}-\mathrm{z}$ e $\mathrm{y}-\mathrm{z}$ e larguras de feixe a meia potência (HPBW) iguais a $92^{\circ}$ e $104^{\circ}$, respectivamente. Para a simulação no CST, o ganho da antena foi igual a 2,16 dBi nas duas direções e uma largura de feixe a meia potência de $94^{\circ}$ para o plano $x-z$ e de $104^{\circ}$ no plano $\mathrm{y}-\mathrm{z}$.

O comportamento da radiação do arranjo 1x2 em função do ganho, pode ser visto na Fig. 4(b). Pode-se observar que o arranjo tornou a antena mais diretiva, ou seja, diminuiu a largura de feixe a meia potência e aumentou o ganho. Para a simulação no HFSS, o arranjo apresentou um ganho máximo de $2,97 \mathrm{dBi}$, para as duas direções, e uma largura de banda a meia potência de $88^{\circ}$ no plano $\mathrm{x}-\mathrm{z}$ e de $50^{\circ}$ no plano y-z. Olhando para a simulação no CST, o ganho máximo foi de 4,7 no plano x-z, com
HPBW de $84^{\circ}$, e no plano y-z o ganho máximo foi de $4,28 \mathrm{dBi}$ com $50^{\circ}$ de HPBW.

De acordo com a Fig. 4(c) nota-se que o arranjo 1x4, assim como os outros, tem sua radiação direcional e tem seu ganho máximo na direção de Broadside. Na simulação do HFSS, o arranjo apresentou um ganho de $6,46 \mathrm{dBi}$ nos planos x-z e y-z, e larguras de feixe a meia potência iguais a $87^{\circ}$ e $26^{\circ}$, respectivamente. Para a simulação no CST, o ganho da antena foi igual a $6,06 \mathrm{dBi}$ nas duas direções e uma largura de feixe a meia potência de $96^{\circ}$ para o plano x-z e de $26^{\circ}$ para o plano y-z.

A Tabela II resume os parâmetros extraídos dos diagramas de radiação.

TABLE II. COMPARAÇÃO DOS RESULTADOS SIMULADOS DO DIAGRAMA DE RADIAÇÃO

\begin{tabular}{|c|c|c|c|c|}
\hline \multirow[t]{2}{*}{ Antena } & \multirow[t]{2}{*}{ Resultado } & \multirow{2}{*}{$\begin{array}{l}\text { Ganho } \\
\text { máximo } \\
\text { (dBi) }\end{array}$} & \multicolumn{2}{|c|}{$\begin{array}{l}\text { Largura de feixe a meia } \\
\text { potência }\end{array}$} \\
\hline & & & Plano $x-z$ & Plano y-z \\
\hline \multirow{2}{*}{$\begin{array}{l}\text { Arranjo } \\
\quad 1 \times 1\end{array}$} & HFSS & 0,00 & $92^{\circ}$ & $104^{\circ}$ \\
\hline & CST & 2,16 & $94^{\circ}$ & $104^{\circ}$ \\
\hline \multirow{2}{*}{$\begin{array}{l}\text { Arranjo } \\
1 \times 2\end{array}$} & HFSS & 2,97 & $88^{\circ}$ & $50^{\circ}$ \\
\hline & CST & 4,70 & $84^{\circ}$ & $50^{\circ}$ \\
\hline \multirow{2}{*}{$\begin{array}{l}\text { Arranjo } \\
1 \times 4\end{array}$} & HFSS & 6,34 & $91^{\circ}$ & $26^{\circ}$ \\
\hline & CST & 6,06 & $96^{\circ}$ & $26^{\circ}$ \\
\hline
\end{tabular}

A Fig. 5 apresenta os diagramas de radiação em 3D das antenas obtidos no HFSS.

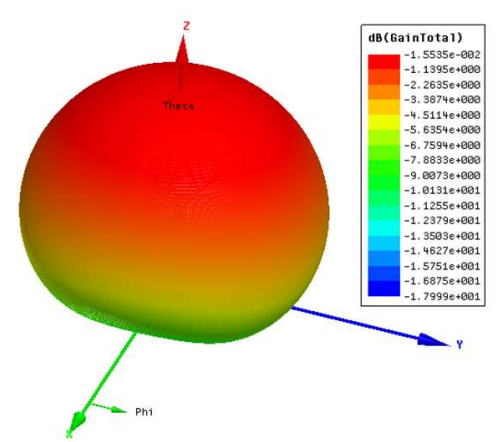

(a)

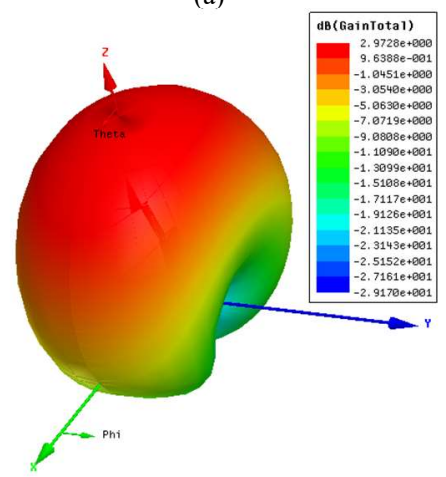

(b) 


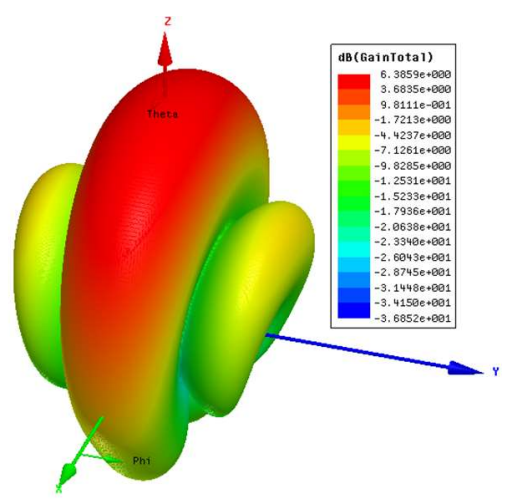

(c)

Fig. 5. Diagramas de radiação simulados 3D: (a) Arranjo 1x1; (b) Arranjo 1x2; e (c) Arranjo $1 \times 4$

Outro parâmetro que foi analisado para as antenas propostas, foi a densidade de corrente, que foi obtida no software HFSS para a frequência de ressonância da antena, a Fig. 6 mostra como se comporta essa densidade. É notável que a corrente está com intensidade maior na parte superior dos patches. E nos arranjos a energia é dividida igualmente entre os elementos ressoadores.

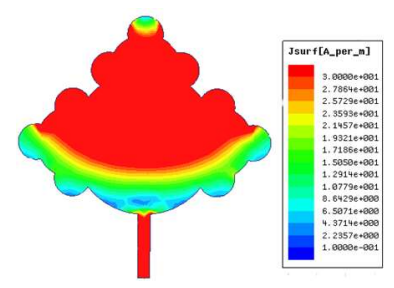

(a)

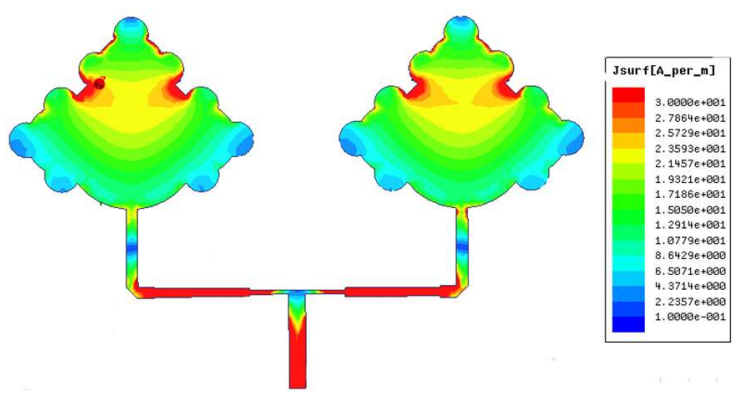

(b)

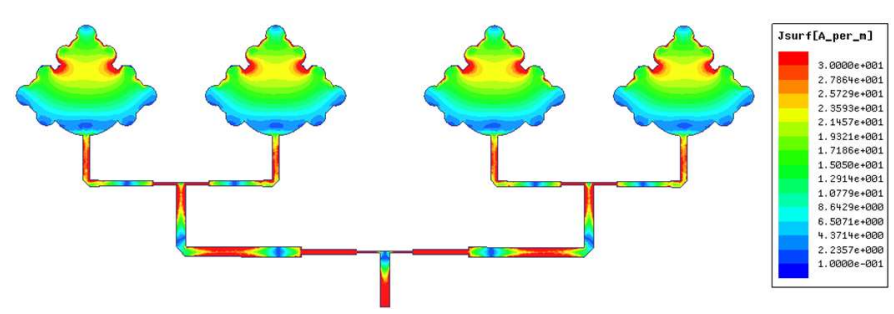

(c)

Fig. 6. Distribuição de densidade de corrente: (a) Arranjo 1x1; (b) Arranjo 1x2; e (c) Arranjo $1 x 4$.

\section{CONCLUSÕES}

Neste trabalho foram realizadas investigações experimentais e teóricas das propriedades de antenas em arranjos planares com patch fractal. Todos os resultados obtidos foram gerados por simulações eletromagnéticas utilizando o método WCIP e os softwares comerciais Ansoft HFSS e o CST Studio. Arranjos de antenas com patch em fractal de tetra círculo foram projetados e construídos, para aplicações na frequência de bluetooth e WI-FI. Para todas as estruturas foram obtidas as curvas de perda de retorno em função da frequência, os diagramas de radiação em 2D e 3D e densidade de corrente no patch. Os resultados obtidos, mostram uma boa coerência da medição com as simulações, e demonstram que com o aumento dos elementos no arranjo, o ganho aumenta e a largura de feixe a meia potência diminui, tornando a estrutura mais diretiva.

\section{AGRADECIMENTOS}

Os autores agradecem o apoio parcial recebido do INCT-CSF, CNPq, CAPES, UFERSA e UFRN.

\section{REFERÊNCIAS}

[1] M. J. Duarte, V. P. Silva Neto e A. G. D’Assunção. "Synthesis and Mechanical Reconfiguration of Ground Plane Tilted Microstrip Antennas Based on Tetra-Circle Fractals". J. Microw. Optoelectron. Electromagn. Appl. [online]. 2020, vol.19, n.2 [cited 2020-06-22], pp.228-241.

[2] C. X. Mao, S. Gao e Y. Wang, "Broadband High-Gain Beam-Scanning Antenna Array for Millimeter-Wave Applications", in IEEE Transactions on Antennas and Propagation, v. 65, no. 9, p. 4864-4868, Set. 2017.

[3] P. Kakaria e R. Nema, "Review and survey of compact and broadband Microstrip Patch Antenna," 2014 International Conference on Advances in Engineering \& Technology Research (ICAETR - 2014), Unnao, 2014, pp. $1-5$.

[4] C. A. Balanis, “Antenna Theory - Analysis and Design”. Harper \& Row, Publishers, New York, 1982.

[5] P. S. Hall e J. R. James, "Survey of design techniques for flat profile microwave antennas and arrays," in Radio and Electronic Engineer, vol. 48, no. 11, pp. 549-565, November 1978.

[6] S. S. Khade e P. D. Bire, "Fractal antenna for wireless applications," 2017 6th International Conference on Reliability, Infocom Technologies and Optimization (Trends and Future Directions) (ICRITO), Noida, India, 2017, pp. 412-415.

[7] B. Singh, N. Sarwade e K. P. Ray, "Compact series FED tapered antenna array using unequal rectangular microstrip antenna elements", in Microwave and Optical Technology Letters, v. 59, no. 8, p. 1856-1861, Dez. 2016.

[8] M. Rabbani e H. Ghafouri-Shiraz, "Evaluation of Gain Enhancement in Improved Size Microstrip Antenna Arrays for Millimetre-wave Applications", in AEU - International Journal of Electronics and Communications. v.81, p.105-113, abriu/julho. 2017.

[9] V. P. Silva Neto, M. J. Duarte, J. K. A. Nogueira e A. G. D’Assunção, "Design of Microstrip Monopoles for Broadband Systems Using an Iterative Wave Formulation", International Microwave and Optoelectronics Conference, Águas de Lindóia, Brazil, 2017.

[10] V. P. Silva Neto, J. K. A. Nogueira e A. G. D’Assunção, "Analysis of band pass filter printed on isotropic and anisotropic substrates by the WCIP method," International Microwave and Optoelectronics Conference, Porto de Galinhos, Brazil, 2015.

[11] V. P. Silva Neto, M. J. Duarte, M. R. M. L. Alburquerque e A. G. D’Assunção, "Analysis and design of fractal-like circular patch elements for miniaturized and stable FSSs," International Microwave and Optoelectronics Conference, Porto de Galinhos, Brazil, 2015.

[12] P. B. C. Medeiros, V. P. Silva Neto e A. G. D’Assunção, “Compact and stable design of FSS with radial slit circular elements using an iterative method," Microwave and Optical Technology Letters, vol. 57, pp. 729733, 2015. 